\title{
Discordant CSF/plasma HIV-1 RNA in patients with unexplained low-level viraemia
}

\author{
Sam Nightingale ${ }^{1,2,3} \cdot$ Anna Maria Geretti $^{1} \cdot$ Apostolos Beloukas $^{1} \cdot$ Martin Fisher $^{4}$. \\ Alan Winston ${ }^{5} \cdot$ Laura Else $^{2} \cdot$ Mark Nelson $^{6} \cdot$ Stephen Taylor $^{7} \cdot$ Andrew Ustianowski $^{8}$. \\ Jonathan Ainsworth ${ }^{9} \cdot$ Richard Gilson $^{10}$ • Lewis Haddow ${ }^{10}$ • Edmund Ong ${ }^{11}$. \\ Victoria Watson ${ }^{2}$. Clifford Leen ${ }^{12}$ • Jane Minton ${ }^{13}$ • Frank Post ${ }^{14}$. \\ Munir Pirmohamed $^{2} \cdot$ Tom Solomon ${ }^{1,15} \cdot$ Saye Khoo ${ }^{2,3}$
}

Received: 27 October 2015 /Revised: 4 April 2016/Accepted: 17 April 2016 /Published online: 18 May 2016

(C) The Author(s) 2016. This article is published with open access at Springerlink.com

\begin{abstract}
The central nervous system has been proposed as a sanctuary site where HIV can escape antiretroviral control and develop drug resistance. HIV-1 RNA can be at higher levels in CSF than plasma, termed CSF/plasma discordance. We aimed to examine whether discordance in CSF is associated with low level viraemia (LLV) in blood. In this MRC-funded multicentre study, we prospectively recruited patients with LLV, defined as one or more episode of unexplained plasma HIV-1 RNA within 12 months, and undertook CSF examination. Separately, we prospectively collected CSF from patients undergoing lumbar puncture for a clinical indication. Patients with durable suppression of viraemia and no evidence of CNS infection were identified as controls from this group. Factors associated with CSF/ plasma HIV-1 discordance overall were examined. One hundred fifty-three patients were recruited across 13 sites; 40 with LLV and 113 undergoing clinical lumbar puncture. Seven of the
\end{abstract}

Part of this data presented at CROI, Boston, USA, 6th March 2014.

Sam Nightingale

s.nightingale@liv.ac.uk

Institute of Infection and Global Health, University of Liverpool, The Ronald Ross Building, 8 West Derby Street, Liverpool L69 7BE, UK

2 Department of Molecular and Clinical Pharmacology, University of Liverpool, Liverpool, UK

3 Royal Liverpool and Broadgreen University Hospitals NHS Trust, Liverpool, UK

4 Brighton and Sussex University Hospitals NHS Trust, Brighton, UK

5 St Marys' Hospital, Imperial College Healthcare NHS Trust, London, UK

6 St Stephen's AIDS Research Trust and Chelsea and Westminster Hospital NHS Foundation Trust, London, UK
$40(18 \%)$ patients with LLV had CSF/plasma discordance, which was significantly more than $0 / 43(0 \%)$ with durable suppression in blood from the clinical group $(p=0.005)$. Resistance associated mutations were shown in six CSF samples from discordant patients with LLV (one had insufficient sample for testing), which affected antiretroviral therapy at sampling in five. Overall discordance was present in 20/153 (13\%) and was associated with nadir CD4 but not antiretroviral concentrations in plasma or CSF. CSF/plasma discordance is observed in patients with LLV and is associated with antiretroviral resistance associated mutations in CSF. The implications for clinical practice require further investigation.

Keywords HIV · Cerebrospinal fluid · Antiretroviral agents · Drug resistance $\cdot$ Viral $\cdot$ Central nervous system

7 Birmingham Heartlands Hospital, Heart of England NHS Foundation Trust, Birmingham, UK

8 North Manchester General Hospital, Pennine Acute Hospitals NHS Trust, Manchester, UK

9 North Middlesex University Hospital NHS Trust, London, UK

10 Research Department of Infection and Population Health, University College London, London, UK

11 Victoria Royal Infirmary, Newcastle upon Tyne Hospitals NHS Trust, Newcastle, UK

12 Royal Infirmary of Edinburgh, NHS Lothian, Edinburgh, UK

13 Leeds General Infirmary, Leeds Teaching Hospitals NHS Trust, Leeds, UK

14 Kings College Hospital NHS Foundation Trust, London, UK

15 Walton Centre for Neurology and Neurosurgery, Liverpool, UK 


\section{Introduction}

The central nervous system (CNS) has been proposed as a sanctuary site where HIV-1 replication may continue during stable antiretroviral therapy (ART). Concentrations of many antiretrovirals (ARVs) in cerebrospinal fluid (CSF) fall below the minimum inhibitory concentration for wild type virus, and there is large inter-individual variability in CSF penetration (Letendre et al. 2010b; Vissers et al. 2010; Best et al. 2012; Peluso et al. 2012; Ciccarelli et al. 2013; Cusini et al. 2013). ARVs may also have reduced activity in some of the cell populations that are susceptible to HIV infection in the CNS (Aquaro et al. 2002; Gray et al. 2013). HIV-1 RNA can be found at higher concentrations in CSF than in plasma in some patients (Eden et al. 2010; Rawson et al. 2012), termed CSF/ plasma discordance, which may result in the compartmentalised detection of drug-resistant variants in CSF (Cunningham et al. 2000; Venturi et al. 2000; Strain et al. 2005).

Unexplained intermittent or persistent low-level viraemia (LLV) in plasma is a common clinical problem, occurring in up to a quarter of ART-treated patients (Doyle and Geretti 2012). The source of this virus is not clear and the clinical significance of LLV is debated (Doyle and Geretti 2012; Doyle et al. 2012). Whether the CNS can act as a sanctuary site for HIV-1 infection in these patients is not known. In this MRC-funded multicentre prospective study, we aimed to investigate the occurrence of $\mathrm{CSF} /$ plasma discordance in patients with LLV compared to patients with durable suppression of HIV-1 RNA in blood. We also aimed to investigate factors associated with CSF/plasma discordance, including ARV concentrations and resistance-associated mutations (RAMs) in the CSF.

\section{Methods}

\section{Study population}

We prospectively recruited HIV-1 positive adults ( $\geq 18$ years) receiving ART from 13 centres in the UK. Written informed consent was obtained from all participants. The study was approved by the North Wales Research Ethics Committee (Central and East).

Patients were recruited in two groups. In the first group, patients with LLV underwent lumbar puncture (LP) for research purposes. LLV was defined as at least one episode of unexplained plasma HIV-1 RNA viraemia above detection threshold (40 copies $/ \mathrm{ml}$ ) within the previous 12 months. No upper limit was specified. We included patients with blips and persistent LLV. We excluded those with an alternative explanation for virological non-suppression such as adherence
$<95 \%$ on self-report or recent commencement of ART. Recent modification of ART was not an exclusion.

It was not considered ethically justifiable to perform research LP on aviraemic controls without neurological symptoms; therefore, we prospectively recruited patients undergoing LP for a clinical indication and collected extra CSF at the time of LP. From this group, we identified patients with durable suppression of HIV-1 RNA detection limits ( 40 copies $/ \mathrm{ml}$ ) over the past year and no evidence of CNS infection to act as controls for those with LLV.

In a separate analysis, we examined factors associated with discordance in the two groups combined, including patients in the clinical group that were not durably suppressed or had evidence of CNS infection.

\section{Laboratory testing}

Virology testing and quantification of drug concentrations were performed centrally at the University of Liverpool. CSF cell count, protein and glucose measurements and microbiological investigations were performed by routine methods at the centres of care. In the LLV group, CSF cell count was performed locally at the discretion of the treating physician.

\section{Virology testing}

HIV-1 RNA was measured centrally in simultaneous plasma and CSF samples by the Abbott RealTime HIV-1 assay (Maidenhead, UK) with a lower limit of quantification of 40 copies/ml, as previously described (Garcia-Diaz et al. 2013). CSF/plasma discordance was defined as CSF HIV-1 RNA levels $>0.5 \log _{10}$ higher than those in plasma based on criteria from other studies (Rawson et al. 2012), and our own work showing raised CSF inflammatory cytokines at this degree of discordance (Nightingale et al. 2016). Discordant samples underwent Sanger (population) sequencing of reverse transcriptase (RT, amino acids 1 to 335), protease (amino acids 1 to 99 ) and integrase (amino acid 1 to 288) to detect RAMs, as previously described (Stockdale et al. 2015).

\section{Drug concentrations}

ARV concentrations were determined in CSF and plasma by liquid chromatography-tandem mass spectrometry (LC-MS/ MS). Drugs were extracted by protein precipitation (plasma) or directly injected (CSF). Standard curves were made up in artificial CSF (Harvard Apparatus, Holliston, MA, USA), and matrix effects assessed by post column injection. Assays were validated against repeated runs of quality controls (inter and intra-assay variability) and external quality assurance standards.

The revised CNS penetration effectiveness (CPE) score (2010) was calculated by assigning a predefined number of 
points to each component of the ART regimen, as proposed by Letendre et al. (Letendre et al. 2008, 2010a). Medication adherence was assessed using the modified medication adherence self report inventory (MASRI) questionnaire at the time of LP.

\section{Cognitive screening tools}

In order to screen for issues related to cognitive impairment, patients with LLV underwent assessment with the following tools. Symptoms of cognitive difficulties were assessed by asking patients to answer whether they had problems with memory, reasoning and attention, as described by Simioni et al. (Simioni et al. 2010), and used in international guidelines (European AIDS Clinical Society 2014) [21] [21] [20] ${ }^{20}$. Patients were considered to have cognitive symptoms when answering "yes, definitely" on at least one of the three questions. Patients were also screened for cognitive impairment using the International HIV Dementia scale (Zipursky et al. 2013). This scale uses three brief tests to assess motor speed, psychomotor speed and memory-recall. A score of less than ten has been considered potentially abnormal (Sacktor et al. 2005). Functional impairment was measured using the Instrumental Activities of Daily Living scale (Heaton et al. 2004; Mind exchange 2013). The maximum score of eight means no impairment in the following activities: telephoning, shopping, preparing food, housekeeping, laundry, travel, medications and finances. A score of seven or less indicates potential functional impairment. Mood disorders were assessed using the Hospital Anxiety and Depression scale, addressing depressive (HAD-D) and anxious (HAD-A) symptoms separately. Patients were considered depressed or anxious if the HAD-D or HAD-A subscale score was at least ten out of 21 (Savard et al. 1998, 1999). Cognitive testing was not performed in the clinical group as this could be confounded by intercurrent illness.

\section{Statistical analysis}

Mann-Whitney $U$ test was used to compare continuous nonparametric variables. Fisher's exact and chi-squared tests were used for categorical data. The geometric mean of $\log _{10}$ ARV concentrations in plasma and CSF was compared between discordant and non-discordant patients with Student's $t$ test and also combined with time post dose in a multivariate logistic regression analysis using the forced entry method. A $p$ value of 0.05 was used to determine statistical significance. When correlating CSF and plasma HIV-1 RNA, levels below detection were assigned an arbitrary mid-point value between zero and the $95 \%$ detection rate. All analyses were performed using SPSS v22.

\section{Results}

\section{Study population}

A total of 153 ART-treated patients were recruited; 40 patients with LLV undergoing LP for research purposes and 113 with clinically indicated LP.

In the 40 patients with LLV, plasma HIV-1 RNA had been measured a median of five times (IQR 4,6 ) in the previous 12 months. HIV-1 RNA had been detected $>50$ copies $/ \mathrm{ml}$ on 117 of 198 occasions; median HIV-1 RNA levels during viraemic episodes was 92 copies/ml (IQR 59, 179). Eight patients $(20 \%)$ reported cognitive symptoms on questionnaire. One patient $(3 \%)$ had a score below ten on the International HIV Dementia scale and one other patient had a score below eight on the Instrumental Activities of Daily Living scale. Ten patients $(25 \%)$ were anxious and two patients $(6 \%)$ were depressed according to the Hospital Anxiety and Depression scale.

In the clinical group, 113 patients underwent LP to investigate cognitive symptoms $(n=50)$, headache $(n=20)$, suspected CNS infection or malignancy $(n=18)$, follow-up of a previous CNS infection $(n=8)$, abnormal neurological examination $(n=7)$, seizure $(n=5)$, neuropathy/ radiculopathy $(n=2)$ and others $(n=3)$. On the basis of clinical findings and results of LP CNS infection was excluded in 92/113 patients $(81 \%)$. Where CNS infection was proven or probable, the diagnoses were neurosyphilis $(n=4)$, cryptococcal meningitis $(n=2)$, cryptococcal immune reconstitution inflammatory syndrome $(n=2)$, herpes simplex virus type-2 meningitis $(n=1)$, viral radiculopathy $(n=1)$, Ebstein-Barr virus in CSF of uncertain significance $(n=4)$ and no diagnosis $(n=7)$. Details of alternative diagnoses in those without CNS infection were not recorded; symptoms were cognitive ( $n=43)$, headache $(n=16)$, suspected CNS infection or malignancy ( $n=12)$, follow-up of a previous CNS infection $(n=4)$, abnormal neurological examination $(n=7)$, seizure $(n=5)$, neuropathy/radiculopathy $(n=2)$ and others $(n=3)$. In this group, 43/113 (38 \%) patients had durable HIV-1 RNA suppression for 12 months and no CNS infection. The remaining 70 either had CNS infection $(n=21)$, were not suppressed $(n=26)$ or were not durably suppressed $(n=23)$.

\section{CSF/plasma HIV-1 RNA discordance}

CSF/plasma discordance was found in 7/40 (18\%) patients with LLV. None of the 43 patients in the clinical group with durable suppression had discordance. The difference in rate of discordance between patients with LLV (18\%) and durable suppression $(0 \%)$ was statistically significant, $p=0.005$ (Fig. 1). In patients with LLV, there was no difference in magnitude or frequency of HIV-1 RNA detection over the previous 12 months between those with $(n=7)$ and those without 


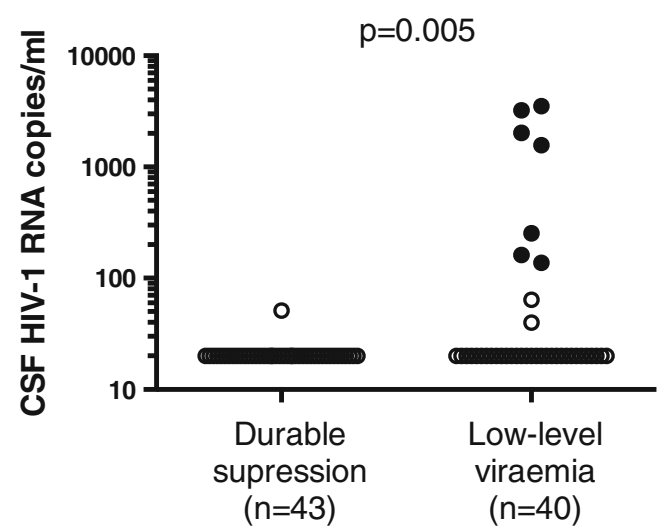

Fig. 1 CSF HIV-1 RNA in 40 patients with intermittent or persistent low-level plasma HIV-1 RNA detection in the last year versus 43 patients from a clinical cohort with durable HIV-1 RNA suppression in the last year. Filled circles represent discordant samples

$(n=33)$ discordance. There were no significant differences in the rate cognitive symptoms, International HIV Dementia Scale results, functional impairment on the Instrumental Activities of Daily Living scale, or tests of anxiety and depression. There was a trend to more frequent raltegravir use in patients with LLV compared to durable suppression (11/40 versus $19 / 133, p=0.060$ ), which likely reflects use of integrase inhibitors to intensify ART in patients with viral persistence.

In the clinical group, discordance was found in 13/113 $(12 \%)$ patients. All discordant patients in this group were either not suppressed in plasma, not durably suppressed in plasma or had CNS infection. When combined with the LLV group, the rate of discordance overall was 20/153 $(13 \%)$. Overall HIV-1 RNA levels in plasma and CSF were directly correlated (Spearman $r=0.51 ; p<0.001$ ). HIV-1 RNA levels were $<50$ copies/ml in $113 / 153$ (74 \%) plasma samples and 115/153 (75\%) CSF samples. In discordant patients median HIV-1 RNA levels were 501 copies/ml (IQR 210, 2016) in CSF versus $<40$ copies/ml (IQR $<40,<40$ ) in plasma. Differences in measured variables among patients with and without CSF/ plasma discordance are shown in Table 1. Patients with discordance showed a lower nadir (but not current) CD4 cell count and a higher CSF white cell count. Lower rates of discordance were observed in white MSM compared with black heterosexuals, which may reflect the higher nadir CD4 cell count in this group (median 173 versus 110 cells $\left./ \mathrm{mm}^{3}, p=0.029\right)$.
Table 1 Univariate analysis of factors associated with discordant HIV-1 RNA levels in paired CSF and plasma samples

\begin{tabular}{|c|c|c|c|}
\hline & \multicolumn{2}{|c|}{$\mathrm{CSF} /$ plasma discordance } & \multirow[t]{2}{*}{$p$ value } \\
\hline & Yes $(n=20)$ & No $(n=133)$ & \\
\hline Age, median years (IQR) & $46(43,50)$ & $46(41,54)$ & 0.913 \\
\hline Gender male, $n(\%)$ & $15(75)$ & $117(88)$ & 0.156 \\
\hline Risk group/ethnicity, $n(\%)$ & & & 0.007 \\
\hline White MSM & $7(35)$ & $80(60)$ & \\
\hline Black heterosexual & $8(40)$ & $17(13)$ & \\
\hline Other & $5(25)$ & $36(27)$ & \\
\hline \multicolumn{4}{|l|}{$\mathrm{CD} 4$, median cells $/ \mathrm{mm}^{3}$ (IQR) } \\
\hline Nadir & $32(21,256)$ & $159(49,281)$ & 0.030 \\
\hline Current & $374(190,613)$ & $464(310,707)$ & 0.163 \\
\hline Years since HIV diagnosis, median (IQR) & $10(7,16)$ & $9(5,16)$ & 0.833 \\
\hline ART at sampling, $n(\%)$ & & & 0.178 \\
\hline $\mathrm{PI} / \mathrm{r}$ based & $14(70)$ & $74(55)$ & \\
\hline NNRTI based & $3(15)$ & $47(35)$ & \\
\hline Other & $3(15)$ & $12(9)$ & \\
\hline CPE, median (IQR) & $7(7,10)$ & $7(6,8)$ & 0.250 \\
\hline Self reported adherence $<95 \%, n(\%)$ & $2(10)$ & $12(9)$ & 1.000 \\
\hline CSF WCC median cells/mm $/ \mathrm{mm}^{3}$ (IQR) & $11(<1,21)$ & $<1(<1,3)$ & 0.018 \\
\hline CNS infection, $n(\%)$ & $3(15)$ & $18(14)$ & 0.740 \\
\hline
\end{tabular}

Risk group and ethnicity were combined due to co-linearity; $89 \%$ of MSM were white. CSF white cell count was tested in $24(60 \%)$ of LLV patients and all patients in the clinical cohort

$M S M$ men who have sex with men, IVDU intravenous drug user, IQR interquartile range, $P I / r$ ritonavir-boosted protease inhibitor, NNRTI non-nucleoside reverse transcriptase inhibitor, NRTI nucleoside/tide reverse transcriptase inhibitor, $R A L$ raltegravir, $M V C$ maraviroc, $C P E$ CNS penetration effectiveness score 2010 


\section{CSF resistance}

CSF samples from $6 / 7$ discordant patients with LLV underwent sequencing for the detection of HIV-1 RAMs (one patient had insufficient CSF for resistance testing). All six samples tested showed RAMs; this affected one or more of the ARVs taken at the time of sampling in 5/6 patients (Table 2). At least one RAM had been previously identified in plasma in all six patients.

In the clinical group, 12/13 CSF samples from discordant patients underwent sequencing (one patient had insufficient CSF for resistance testing). Five CSF samples did not amplify despite repeated attempts and one sample only amplified for the protease gene. Of the six CSF samples successfully tested, all showed RAMs; this affected one or more of the ARVs taken at the time of sampling in all six patients (Table 2). CSF RAMs had been previously identified in plasma in one patient (patient 18).

Resistance testing of plasma virus was attempted in patients 7, 10 and 11. Plasma virus from patients 7 and 10 did not amplify. Plasma from patient 11 showed no NRTI or NNRTI RAMs; the integrase gene did not amplify.

\section{Plasma and CSF pharmacokinetics}

ARV concentrations in plasma and CSF are shown in Table 3. No significant differences were observed between those with or without discordance for any ARVs tested in either CSF or plasma. There were also no significant differences in ARV concentrations between those with LLV versus durable suppression. No associations were observed with CPE score or ART use, either for individual ARVs or drug class of regimen (Table 1).

Table 2 CSF ARV resistance associated mutations (RAMs) in 20 patients with CSF/plasma discordance

\begin{tabular}{|c|c|c|c|c|c|c|c|}
\hline \multicolumn{4}{|c|}{ HIV-1 RNA copies/ml } & \multicolumn{4}{|c|}{ CSF resistance associated mutations } \\
\hline Patient ID & Plasma & $\mathrm{CSF}$ & ART at sampling & PI & NRTI & NNRTI & INT \\
\hline \multicolumn{8}{|c|}{ Low-level viraemia group } \\
\hline 1 & $<40$ & 138 & TDF/FTC/DRV/r & - & M184I & - & - \\
\hline 2 & $<40$ & 254 & $\mathrm{EFZ/TDF/FTC}$ & Insufficient sample & & & \\
\hline 3 & 48 & 2028 & $\mathrm{DRV} / \mathrm{r} / \mathrm{TDF} / \mathrm{RAL}$ & None & $\begin{array}{l}D 67 N, K 70 R, L 74 V, M 184 V \\
\quad T 215 Y, K 219 E\end{array}$ & L100IL, K103KN & T66I, Y143C \\
\hline 4 & 78 & 3234 & $\mathrm{DRV} / \mathrm{r} / \mathrm{MVC} / \mathrm{ETR}$ & None & $A 62 \mathrm{~V}, \mathrm{~K} 65 \mathrm{R}, \mathrm{M184V}$ & None & - \\
\hline 5 & 88 & 1569 & TDF/FTC/DRV/r & None & M184I & None & - \\
\hline 6 & $<40$ & 162 & TDF/FTC/DRV/r & V82A & $D 67 N, M 184 V, T 215 Y, K 219 Q$ & V108I & - \\
\hline 7 & 258 & 3518 & $\mathrm{ABC} / 3 \mathrm{TC} / \mathrm{DRV} / \mathrm{r}$ & None & L74LV, M184V & None & - \\
\hline \multicolumn{8}{|c|}{ Clinical cohort } \\
\hline 8 & 40 & 1038 & TDF/FTC/DRV/r & None & Incomplete testing & & \\
\hline 9 & 52 & 1231 & TDF/FTC/RAL/MVC & Did not amplify & & & \\
\hline 10 & 3443 & 13088 & 3TC/AZT/EFZ & - & None & V108I, E138A & \\
\hline 11 & 10817 & 63010 & TDF/FTC/RAL & - & None & None & L74I \\
\hline 12 & $<40$ & 400 & $\mathrm{ABC} / 3 \mathrm{TC} / \mathrm{DRV} / \mathrm{r} / \mathrm{RAL}$ & Did not amplify & & & \\
\hline 13 & $<40$ & 422 & TDF/FTC/ATZ/r & - & T69S, M184I & - & - \\
\hline 14 & $<40$ & 335 & TDF/FTC/DRV/r & Did not amplify & & & \\
\hline 15 & $<40$ & 162 & 3TC/AZT/DRV/r/MVC/ETR & Insufficient sample & & & \\
\hline 16 & $<40$ & 129 & $\mathrm{DRV} / \mathrm{r}$ & Did not amplify & & & \\
\hline 17 & $<40$ & 579 & $\mathrm{ATZ} / \mathrm{r} / \mathrm{TDF} / \mathrm{FTC}$ & V82AV & M184V, T215I & - & - \\
\hline 18 & $<40$ & 1981 & $\mathrm{DRV} / \mathrm{r} / \mathrm{RAL} / \mathrm{MVC} / 3 \mathrm{TC}$ & None & $M 184 V$ & V108I & $\mathrm{N155H}$ \\
\hline 19 & $<40$ & 195 & EFZ/TDF/FTC & Did not amplify & & & \\
\hline 20 & $<40$ & 405 & LPV/r/AZT/RAL & None & None & None & N155H \\
\hline
\end{tabular}

RAMs in bold are to ARVs taken at the time of sampling. RAMs in italics have been previously detected in plasma. Patient 9 showed CCR5 tropic virus in CSF

$P I$ protease inhibitor, $N R T I$ nucleoside/tide reverse transcriptase inhibitor, NNRTI non-nucleoside reverse transcriptase inhibitor, INT integrase inhibitor, $A T Z$ atazanavir, $D R V$ darunavir, $L P V$ lopinavir, ETV etravirine, $M V C$ maraviroc, $N V P$ nevirapine, $R A L$ raltegravir, $R P V$ rilpivarine, $r$ ritonavir, $E F Z$ efavirenz, $T D F$ tenofovir, $F T C$ emtricitabine, $3 T C$ lamivudine, $A Z T$ zidovudine 
Table 3 Antiretroviral concentrations in CSF and plasma

\begin{tabular}{|c|c|c|c|}
\hline & \multirow[t]{2}{*}{$n$} & \multicolumn{2}{|c|}{ Drug concentration (ng/ml) } \\
\hline & & Geo mean & $95 \% \mathrm{CI}$ \\
\hline \multicolumn{4}{|l|}{ Plasma } \\
\hline Tenofovir & 62 & 89.5 & $73.1-110.7$ \\
\hline Emtricitabine & 65 & 187.9 & $136.5-264.9$ \\
\hline Lamivudine & 24 & 383.7 & $225.4-688.7$ \\
\hline Darunavir & 55 & 2971.7 & $2393.3-3715.4$ \\
\hline Atazanavir & 19 & 729.5 & $418.8-1339.7$ \\
\hline Lopinavir & 3 & 8570.4 & $7798.3-9440.6$ \\
\hline Ritonavir & 15 & 116.1 & $55.7-277.3$ \\
\hline Efavirenz & 27 & 1741.8 & $1349.0-2269.9$ \\
\hline Nevirapine & 10 & 4226.7 & $2691.5-6807.7$ \\
\hline Etravirine & 4 & 269.2 & $64.4-1836.5$ \\
\hline Rilpivarine & 4 & 68.1 & $36.0-144.2$ \\
\hline Maraviroc & 8 & 172.2 & $88.9-367.3$ \\
\hline Raltegravir & 11 & 413.9 & $174.1-1135.0$ \\
\hline \multicolumn{4}{|l|}{ CSF } \\
\hline Darunavir & 54 & 35.2 & $27.8-45.4$ \\
\hline Atazanavir & 20 & 6.4 & $4.1-11.5$ \\
\hline Lopinavir & 3 & 9.6 & $4.1-37.3$ \\
\hline Efavirenz & 26 & 14.7 & $11.4-19.4$ \\
\hline Nevirapine & 12 & 1559.6 & $1127.2-2197.9$ \\
\hline Maraviroc & 9 & 6.5 & $2.75-31.6$ \\
\hline Raltegravir & 14 & 25.9 & $18.1-38.7$ \\
\hline
\end{tabular}

Geometric mean and $95 \%$ confidence interval for drug concentrations in plasma and CSF. No significant associations were observed with CSF/ plasma discordance

\section{Discussion}

We observed CSF/plasma discordance in $18 \%$ of patients undergoing a research-based LP for intermittent or continuous LLV. No discordance was observed in patients with durably suppressed plasma viral load undergoing clinically indicated LP. This suggests that a significant proportion of HIV-positive patients with LLV despite ART may have suboptimal control of virus replication within the CNS. CSF examination of discordant patients with LLV revealed RAMs in all samples tested, which related to ART taken at the time of sampling in all but one. Of note, in these patients, plasma viraemia was generally too low to perform resistance testing in blood.

One interpretation of these findings could be that ongoing replication of HIV in a CNS sanctuary site results in seeding to plasma leading to LLV. Alternatively, the observation of LLV and CSF/plasma discordance may represent two coinciding aspects of overall suboptimal virological suppression. It is of interest that in all cases at least part of the resistance detected in CSF virus was consistent with the previous history of failure and older resistance tests in plasma. This, combined with the observed association of discordance with low nadir CD4, suggests that advanced immune suppression may allow the establishment of a viral reservoir in the CNS that may be only partially responsive to ART. This virus may then continue to evolve and develop resistance to some ARVs, for example raltegravir, and thus become progressively less susceptible to suppression than virus in peripheral blood. LLV with a genetically different virus may originate from a similar process of incomplete suppression at other sites such as lymphoid tissue, where drug penetration and activity may also vary (Fletcher et al. 2014). Intermittent reseeding of virus into the CNS during periods of systemic replication due to another cause (e.g. non-declared poor adherence) may also establish compartmentalised CNS infection (Gisslen et al. 2005; Eden et al. 2010). Regardless of the origin of CSF virus, targeting LP to patients with unexplained blips or persistent LLV in plasma may represent a useful strategy in clinical practice and warrants further investigation. Altering ART based on the resistance profile of CSF variants has led to clinical improvement and decrease in CSF HIV-1 RNA in case series (Canestri et al. 2010; Peluso et al. 2012) and has been adopted by guidelines as best practice (Mind exchange 2013; Williams et al. 2014). In addition, these findings add to the evidence suggesting the CNS compartment must be considered in studies aiming to eradicate HIV from the periphery.

Screening for cognitive impairment in the LLV group with a cognitive symptoms questionnaire and International HIVDementia Scale did not reveal associations with CSF/plasma discordance. The numbers are small and the screening methods used are insensitive for milder cognitive disorders (Haddow et al. 2013); nevertheless, these data suggest that discordant patients were relatively asymptomatic and cognitive deficits were not marked. Whether discordance in this group has implications for cognitive dysfunction in the longer term requires clarification in longitudinal studies.

$\mathrm{CSF} /$ plasma discordance was present in $12 \%$ of patients undergoing clinically indicated LP. This rate of discordance is similar to other reports from clinical settings (Eden et al. 2010; Rawson et al. 2012). Studies where LP was performed for research purposes in the absence of clinical indications have reported lower rates of discordance (Heaton et al. 2010). CSF/ plasma discordance overall was not associated with ART at sampling, CPE score or ARV concentrations in plasma or CSF, but was associated with nadir CD4 cell count. This suggests the presence of HIV in the CNS relates to a longer duration of viral replication and a lower immune status, consistent with the hypothesis of CNS viral persistence discussed above. In cross sectional studies, nadir CD4 has been consistently associated with cognitive impairment (Robertson et al. 2007; Heaton et al. 2010). It has not been clear whether this 
association is due to a legacy effect of CNS damage sustained prior to ART initiation or whether it represents an ongoing CNS inflammatory process set up during advanced immunosuppression. In our study, all discordant CSF samples successfully tested showed RAMs. Overall RAMs were demonstrated in 12 CSF samples including NRTI RAMs in eight of ten on NRTIs at sampling and raltegravir RAMs in all four patients on raltegravir at sampling. Other work on this cohort has demonstrated raised inflammatory cytokines in CSF of patients with discordance, even at relatively low levels (Nightingale et al. 2016).

There are a number of limitations to our study. Selecting controls from patients undergoing clinically indicated LP was not ideal; however, as clinical cohorts have shown higher rates of discordance than those undergoing LP for research purposes, this risked producing a false negative rather than false positive result. We assessed adherence by self-report which may not be reliable in all patients. Of note, there were no observed differences in measured plasma ARV concentrations between those with and without discordance suggesting that adherence was similar between groups, at least in terms of very recent ART use. Differences in the rate of viral decay between compartments in those recently starting or changing ART could have led us to identify some with discordance that did not have true compartmentalisation (Schnell et al. 2009). Discordant patients in the LLV group had been on ART for many years; however, recent ART modification was not an exclusion and could have contributed. In the clinical group, we did not routinely collect details of ART history and cannot exclude that this was the source of discordance in some patients. Our analysis of factors related to discordance involved grouping the LLV group with the more heterogeneous clinical group, some of which were not suppressed and/or had CNS infection. This group is reflective of patients undergoing CSF examination in the UK clinical practice and may be more transferable to the clinical environment. Indeed studies recruiting asymptomatic patients for research LP, such as the CHARTER cohort in the USA, found few cases of discordance despite sampling large patient populations (Heaton et al. 2010). Our ability to find associations of discordance with ARV concentrations in CSF and plasma was limited by few measurements for some drugs. In addition, ARV concentrations in CSF may not be the most important factor determining effectiveness of ART in the CNS (Calcagno et al. 2014), in particular ARV concentrations in CSF may not reflect levels within perivascular macrophages, the critical target of HIV in the brain (McArthur et al. 2010). Our finding of no link between CPE score and discordance should also be interpreted with caution as our study was not designed to look for such an association and may be confounded by prescriber bias, whereby patients with CNS problems are more likely to receive an ART regimen with a higher CPE score (Garvey et al. 2011).

Acknowledgments We thank all investigators and research staff that worked on the PARTITION study. We acknowledge infrastructural support for bioanalysis from the Liverpool Biomedical Research Centre funded by Liverpool Health Partners. We also acknowledge the use of the NIHR/Wellcome Trust Clinical Research Facility at King's College Hospital.

Compliance with ethical standards Written informed consent was obtained from all participants. The study was approved by the North Wales Research Ethics Committee (Central and East).

Funding SN is a MRC Clinical Training Fellow supported by the North West England Medical Research Council Fellowship Scheme in Clinical Pharmacology and Therapeutics, which is funded by the Medical Research Council (grant number G1000417/94909), ICON, GlaxoSmithKline, AstraZeneca and the Medicines Evaluation Unit. SN also received a research award to support this work from the British HIV Association.

Conflicts of interest The authors declare that they have no competing interest.

Open Access This article is distributed under the terms of the Creative Commons Attribution 4.0 International License (http:// creativecommons.org/licenses/by/4.0/), which permits unrestricted use, distribution, and reproduction in any medium, provided you give appropriate credit to the original author(s) and the source, provide a link to the Creative Commons license, and indicate if changes were made.

\section{References}

Aquaro S, Calio R, Balzarini J, Bellocchi MC, Garaci E, Perno CF (2002) Macrophages and HIV infection: therapeutical approaches toward this strategic virus reservoir. Antiviral Res 55(2):209-225

Best BM, Letendre SL, Koopmans P, Rossi SS, Clifford DB, Collier AC, Gelman BB, Marra CM, McArthur JC, McCutchan JA, Morgello S, Simpson DM, Capparelli EV, Ellis RJ, Grant I (2012) Low cerebrospinal fluid concentrations of the nucleotide HIV reverse transcriptase inhibitor, tenofovir. J Acquir Immune Defic Syndr 59(4):376381

Calcagno A, Di Perri G, Bonora S (2014) Pharmacokinetics and pharmacodynamics of antiretrovirals in the central nervous system. Clin Pharmacokinet 53(10):891-906

Canestri A, Lescure FX, Jaureguiberry S, Moulignier A, Amiel C, Marcelin AG, Peytavin G, Tubiana R, Pialoux G, Katlama C (2010) Discordance between cerebral spinal fluid and plasma HIV replication in patients with neurological symptoms who are receiving suppressive antiretroviral therapy. Clin Infect Dis 50(5):773-778

Ciccarelli N, Fabbiani M, Colafigli M, Trecarichi EM, Silveri MC, Cauda R, Murri R, De Luca A, Di Giambenedetto S (2013) Revised central nervous system neuropenetration-effectiveness score is associated with cognitive disorders in HIV-infected patients with controlled plasma viraemia. Antivir Ther 18(2):153-160

Cunningham PH, Smith DG, Satchell C, Cooper DA, Brew B (2000) Evidence for independent development of resistance to HIV-1 reverse transcriptase inhibitors in the cerebrospinal fluid. AIDS 14(13):1949-1954 
Cusini A, Vernazza PL, Yerly S, Decosterd LA, Ledergerber B, Fux CA, Rohrbach J, Widmer N, Hirschel B, Gaudenz R, Cavassini M, Klimkait T, Zenger F, Gutmann C, Opravil M, Gunthard HF (2013) Higher CNS penetration-effectiveness of long-term combination antiretroviral therapy is associated with better HIV-1 viral suppression in cerebrospinal fluid. J Acquir Immune Defic Syndr 62(1):28-35

Doyle T, Geretti AM (2012) Low-level viraemia on HAART: significance and management. Curr Opin Infect Dis 25(1):17-25

Doyle T, Smith C, Vitiello P, Cambiano V, Johnson M, Owen A, Phillips AN, Geretti AM (2012) Plasma HIV-1 RNA detection below 50 copies $/ \mathrm{ml}$ and risk of virologic rebound in patients receiving highly active antiretroviral therapy. Clin Infect Dis 54(5):724-732

Eden A, Fuchs D, Hagberg L, Nilsson S, Spudich S, Svennerholm B, Price RW, Gisslen M (2010) HIV-1 viral escape in cerebrospinal fluid of subjects on suppressive antiretroviral treatment. J Infect Dis 202(12): 1819-1825

European AIDS Clinical Society Guidelines. Version 7.1 November 2014. http://www.eacsociety.org/Portals/0/GUIDELINES/English PDF - Version 7.1.pdf. Date accessed 27/12/2014

Fletcher CV, Staskus K, Wietgrefe SW, Rothenberger M, Reilly C, Chipman JG, Beilman GJ, Khoruts A, Thorkelson A, Schmidt TE, Anderson J, Perkey K, Stevenson M, Perelson AS, Douek DC, Haase AT, Schacker TW (2014) Persistent HIV-1 replication is associated with lower antiretroviral drug concentrations in lymphatic tissues. Proc Natl Acad Sci U S A 111(6):2307-2312

Garcia-Diaz A, Labbett W, Clewley GS, Guerrero-Ramos A, Geretti AM (2013) Comparative evaluation of the Artus HIV-1 QS-RGQ assay and the Abbott RealTime HIV-1 assay for the quantification of HIV1 RNA in plasma. J Clin Virol 57(1):66-69

Garvey L, Winston A, Walsh J, Post F, Porter K, Gazzard B, Fisher M, Leen C, Pillay D, Hill T, Johnson M, Gilson R, Anderson J, Easterbrook P, Bansi L, Orkin C, Ainsworth J, Palfreeman A, Gompels M, Phillips AN, Sabin CA (2011) Antiretroviral therapy CNS penetration and HIV-1-associated CNS disease. Neurology 76(8):693-700

Gisslen M, Rosengren L, Hagberg L, Deeks SG, Price RW (2005) Cerebrospinal fluid signs of neuronal damage after antiretroviral treatment interruption in HIV-1 infection. AIDS Res Ther 2:6

Gray LR, Tachedjian G, Ellett AM, Roche MJ, Cheng WJ, Guillemin GJ, Brew BJ, Turville SG, Wesselingh SL, Gorry PR, Churchill MJ (2013) The NRTIs lamivudine, stavudine and zidovudine have reduced HIV-1 inhibitory activity in astrocytes. PLoS One 8(4):e62196

Haddow LJ, Floyd S, Copas A, Gilson RJ (2013) A systematic review of the screening accuracy of the HIV Dementia Scale and International HIV Dementia Scale. PLoS One 8(4):e61826

Heaton RK, Marcotte TD, Mindt MR, Sadek J, Moore DJ, Bentley H, McCutchan JA, Reicks C, Grant I (2004) The impact of HIVassociated neuropsychological impairment on everyday functioning. J Int Neuropsychol Soc 10(3):317-331

Heaton RK, Clifford DB, Franklin DR Jr, Woods SP, Ake C, Vaida F, Ellis RJ, Letendre SL, Marcotte TD, Atkinson JH, Rivera-Mindt M, Vigil OR, Taylor MJ, Collier AC, Marra CM, Gelman BB, McArthur JC, Morgello S, Simpson DM, McCutchan JA, Abramson I, Gamst A, Fennema-Notestine C, Jernigan TL, Wong J, Grant I (2010) HIV-associated neurocognitive disorders persist in the era of potent antiretroviral therapy: CHARTER Study. Neurology 75(23):2087-2096

Letendre S, Marquie-Beck J, Capparelli E, Best B, Clifford D, Collier AC, Gelman BB, McArthur JC, McCutchan JA, Morgello S, Simpson D, Grant I, Ellis RJ (2008) Validation of the CNS Penetration-Effectiveness rank for quantifying antiretroviral penetration into the central nervous system. Arch Neurol 65(1):65-70

Letendre S, Ellis RJ, Deutsch R, Clifford DB, Marra CM, McCutchan A, Morgello S, Simpson DM, Heaton RK, I. Grant and C. group (2010a) Correlates of time-to-loss-of-viral-response in csf and plasma in the
CHARTER Cohort. Paper \# 430. 17th Conference on Retroviruses and Opportunistic Infections (CROI 2010). San Fransisco, USA

Letendre S, Fitzsimons C, Ellis RJ, Clifford D, Collier AC, Gelman BB, McArthur JC, Vaida F, Heaton RK I. Grant and CHARTER (2010b) Correlates of CSF viral loads in 1,221 volunteers of the CHARTER Cohort. Paper \#172. 17th Conference on Retroviruses and Opportunistic Infections (CROI 2010). San Fransisco, USA

McArthur JC, Steiner J, Sacktor N, Nath A (2010) Human immunodeficiency virus-associated neurocognitive disorders: mind the gap. Ann Neurol 67(6):699-714

Mind exchange group (2013) Assessment, diagnosis, and treatment of HIV-associated neurocognitive disorder: a consensus report of the mind exchange program. Clin Infect Dis 56(7):1004-1017

Nightingale S, Michael BD, Fisher M, Winston A, Nelson M, Taylor S, Ustianowski A, Ainsworth J, Gilson R, Haddow L, Ong E, Leen C, Minton J, Post F, Beloukas A, Borrow R, Pirmohamed M, Geretti AM, Khoo S, Solomon T (2016) CSF/plasma HIV-1 RNA discordance even at low levels is associated with up-regulation of host inflammatory mediators in CSF. Cytokine 83:139-146

Peluso MJ, Ferretti F, Peterson J, Lee E, Fuchs D, Boschini A, Gisslen M, Angoff N, Price RW, Cinque P, Spudich S (2012) Cerebrospinal fluid HIV escape associated with progressive neurologic dysfunction in patients on antiretroviral therapy with well controlled plasma viral load. AIDS 26(14): 1765-1774

Rawson T, Muir D, Mackie NE, Garvey LJ, Everitt A, Winston A (2012) Factors associated with cerebrospinal fluid HIV RNA in HIV infected subjects undergoing lumbar puncture examination in a clinical setting. J Infect 65(3):239-245

Robertson KR, Smurzynski M, Parsons TD, Wu K, Bosch RJ, Wu J, McArthur JC, Collier AC, Evans SR, Ellis RJ (2007) The prevalence and incidence of neurocognitive impairment in the HAART era. AIDS 21(14):1915-1921

Sacktor NC, Wong M, Nakasujja N, Skolasky RL, Selnes OA, Musisi S, Robertson K, McArthur JC, Ronald A, Katabira E (2005) The International HIV Dementia Scale: a new rapid screening test for HIV dementia. AIDS 19(13):1367-1374

Savard J, Laberge B, Gauthier JG, Ivers H, Bergeron MG (1998) Evaluating anxiety and depression in HIV-infected patients. J Pers Assess 71(3):349-367

Savard J, Laberge B, Gauthier JG, Bergeron MG (1999) Screening clinical depression in HIV-seropositive patients using the Hospital Anxiety and Depression Scale. AIDS Behav 3(2):167-175

Schnell G, Spudich S, Harrington P, Price RW, Swanstrom R (2009) Compartmentalized human immunodeficiency virus type 1 originates from long-lived cells in some subjects with HIV-1-associated dementia. PLoS Pathog 5(4):e1000395

Simioni S, Cavassini M, Annoni JM, Rimbault Abraham A, Bourquin I, Schiffer V, Calmy A, Chave JP, Giacobini E, Hirschel B, Du Pasquier RA (2010) Cognitive dysfunction in HIV patients despite long-standing suppression of viremia. AIDS 24(9):1243-1250

Stockdale AJ, Phillips RO, Beloukas A, Appiah LT, Chadwick D, Bhagani S, Bonnett L, Sarfo FS, Dusheiko G, Geretti AM and B. I. i. K. S. G. Hepatitis (2015) Liver fibrosis by transient elastography and virologic outcomes after introduction of tenofovir in lamivudine-experienced adults with HIV and Hepatitis B virus coinfection in Ghana. Clin Infect Dis 61(6):883-891

Strain MC, Letendre S, Pillai SK, Russell T, Ignacio CC, Gunthard HF, Good B, Smith DM, Wolinsky SM, Furtado M, Marquie-Beck J, Durelle J, Grant I, Richman DD, Marcotte T, McCutchan JA, Ellis RJ, Wong JK (2005) Genetic composition of human immunodeficiency virus type 1 in cerebrospinal fluid and blood without treatment and during failing antiretroviral therapy. J Virol 79(3):1772-1788

Venturi G, Catucci M, Romano L, Corsi P, Leoncini F, Valensin PE, Zazzi M (2000) Antiretroviral resistance mutations in human immunodeficiency virus type 1 reverse transcriptase and protease from paired cerebrospinal fluid and plasma samples. J Infect Dis 181(2):740-745 
Vissers M, Stelma FF, Koopmans PP (2010) Could differential virological characteristics account for ongoing viral replication and insidious damage of the brain during HIV 1 infection of the central nervous system? J Clin Virol 49(4):231-238

Williams I, Churchill D, Anderson J, Boffito M, Bower M, Cairns G, Cwynarski K, Edwards S, Fidler S, Fisher M, Freedman A, Geretti AM, Gilleece Y, Horne R, Johnson M, Khoo S, Leen C, Marshall N, Nelson M, Orkin C, Paton N, Phillips A, Post F, Pozniak A, Sabin C, Trevelion R, Ustianowski A, Walsh J, Waters L, Wilkins E, Winston
A, Youle M (2014) British HIV Association guidelines for the treatment of HIV-1-positive adults with antiretroviral therapy 2012 (Updated November 2013. All changed text is cast in yellow highlight.). HIV Med 15(Suppl 1):1-85

Zipursky AR, Gogolishvili D, Rueda S, Brunetta J, Carvalhal A, McCombe JA, Gill MJ, Rachlis A, Rosenes R, Arbess G, Marcotte T, Rourke SB (2013) Evaluation of brief screening tools for neurocognitive impairment in HIV/AIDS: a systematic review of the literature. AIDS 27(15):2385-2401 